\title{
CORRESPONDENCE
}

\section{Response to "Addiction is a social disease: just as tenable as calling it a brain disease"}

\author{
Neuropsychopharmacology (2021) 46:1713-1714; https://doi.org/ \\ 10.1038/s41386-021-01037-y
}

In his commentary, Dr Room asserts that our conceptualization of substance addiction as a brain disease [1] fails to appropriately acknowledge the social aspects of the disorder [2]. He further asserts that addiction could be characterized as a "social disease" just as well as a brain disease, with equal weight given to the environmental and neurobiological factors.

We agree with the importance of social factors in addiction and discuss their role at length in our paper. However, in our opinion, qualitatively distinguishing between a social and biological disorder is untenable. Dr Room argues that addiction could be considered a social disease because the type of addiction varies with environmental circumstance. For example, the prevalence of opioid addiction in the United States increased following changes in the marketing of prescription opioids, while alcohol addiction is less common in Islamic countries where drinking is banned.

It is obvious, however, that the same can be said for most medical conditions: environment has an impact on their prevalence, course, and prognosis. We and others [3] commonly cite Type 2 diabetes as an example. The rates of this disease vary dramatically with social factors such as lifestyle, diet, education level, and availability of processed foods. But it is perhaps more informative to consider cancer, a disease that is even more universally accepted as having a biological etiology, albeit with important environmental modifiers. In the United States, lung cancer peaked in the early 1990s and has since tapered off due to changes in tobacco control [4]. Conversely, in England, melanoma rates have risen, partly due to increases in sunbathing and use of tanning salons [5]. Nonetheless, cancer is rarely considered a "social disease."

Therefore, it may be worth asking: what is a social disease? In the past, "social disease" was a euphemism for sexually transmitted diseases. A PubMed search for "social disease" returns a wide range of results, from Covid-19 infection to leprosy. Without a doubt, social factors are critical to a wide range of diseases. Nevertheless, there is a very little questioning over whether biological processes are operative, and understanding these changes is also what allows us to identify mechanisms that might be amenable to biomedical treatments.

As we point out, this in no way negates the role of social factors, and policy measures that address those are as important as individual-level treatment interventions; as Room [2] points out, this should be reflected in funding opportunities. But we nonetheless foresee the need for medical treatments, including treatments addressing disordered brain mechanisms. Thus, policy strategies to address social factors and medical treatments to address biological factors do not oppose each other.
What is notable is the double standard regarding different levels of analysis in understanding addiction. We can think of no scientist investigating the biological bases of addiction who would globally reject the importance of behavioral, social, or environmental factors, but there are numerous strident rejections of addiction as a brain disease in the scholarly literature. Why is that the case? As scientists, we are all fundamentally empiricists and rather than rejecting any major line of inquiry, a more constructive approach is an ecumenical one, one that does not pit perspectives against one another, but directly considers which levels of analysis most trenchantly reveal the causes of addiction and guide its treatment. Here again is our call for consilience in the science of addiction.

Ultimately, however, acknowledging the disease nature and the biological underpinnings of addiction is also essential because of a human dimension. Acknowledging these underpinnings promotes medicine, not moral judgment, as the way to understand and treat addiction.

\section{FUNDING AND DISCLOSURE}

Supported by the Swedish Research Council Grants 2013-07434, 2019-01138 (MH); Netherlands Organisation for Health Research and Development (ZonMw) under project number 912.14.093 (LJMJV); NIDA and NIAAA intramural research programs (LL; the content is solely the responsibility of the author and does not necessarily represent the official views of the National Institutes of Health); the Peter Boris Chair in Addictions Research, Homewood Research Institute, and the National Institute on Alcohol Abuse and Alcoholism Grants AA025911, AA024930, AA025849, AA027679 (JM; the content is solely the responsibility of the author and does not necessarily represent the official views of the National Institutes of Health).

$\mathrm{MH}$ has received consulting fees, research support or other compensation from Indivior, Camurus, BrainsWay, Aelis Farma, and Janssen Pharmaceuticals. JM is a Principal and Senior Scientist at BEAM Diagnostics, Inc. The other authors declare no competing interests.

\section{AUTHOR CONTRIBUTIONS}

All authors jointly drafted the paper.

\section{ADDITIONAL INFORMATION}

Publisher's note Springer Nature remains neutral with regard to jurisdictional claims in published maps and institutional affiliations. 
Markus Heilig ${ }^{1}$, James Mackillop (iD ${ }^{2,3}$, Diana Martinez ${ }^{4}$, Jürgen Rehm $\mathbb{D}^{5,6,7,8}$, Lorenzo Leggio $\mathbb{1 D}^{9}$ and Louk J. M. J. Vanderschuren (iD ${ }^{10}$

${ }^{1}$ Center for Social and Affective Neuroscience, Department of Biomedical and Clinical Sciences, Linköping University, Linköping, Sweden; ${ }^{2}$ Peter Boris Centre for Addictions Research, McMaster University and St. Joseph's Healthcare Hamilton, Hamilton, ON, Canada; ${ }^{3}$ Homewood Research Institute, Guelph, ON, Canada; ${ }^{4}$ New York State Psychiatric Institute and Columbia University Irving Medical Center, New York, NY, USA; ${ }^{5}$ Institute for Mental Health

Policy Research \& Campbell Family Mental Health Research Institute, Centre for Addiction and Mental Health (CAMH), Toronto, ON, Canada; ${ }^{6}$ Dalla Lana School of Public Health and Department of Psychiatry, University of Toronto (UofT), Toronto, ON, Canada; ${ }^{7}$ Klinische Psychologie \& Psychotherapie, Technische Universität Dresden, Dresden, Germany; ${ }^{8}$ Department of International Health Projects, Institute for Leadership and Health Management, I.M. Sechenov First Moscow State Medical University, Moscow, Russia; ${ }^{9}$ Clinical Psychoneuroendocrinology and Neuropsychopharmacology Section, Translational Addiction Medicine Branch, National Institute on Drug Abuse Intramural Research Program and National Institute on Alcohol Abuse and Alcoholism Division of Intramural Clinical and

Biological Research, National Institutes of Health, Baltimore and Bethesda, MD, USA and ${ }^{10}$ Department of Population Health Sciences, Unit Animals in Science and Society, Faculty of Veterinary Medicine, Utrecht University, Utrecht, the Netherlands Correspondence: Markus Heilig (markus.heilig@liu.se)

\section{REFERENCES}

1. Heilig M, MacKillop J, Martinez D, Rehm J, Leggio L, Vanderschuren LJ. Addiction as a brain disease revised: why it still matters, and the need for consilience. Neuropsychopharmacology. 2021:1-9. https://doi.org/10.1038/s41386-020-00950-y.

2. Room R. "Addiction is a social disease": just as tenable as calling it a brain disease. Neuropsychopharmacology. 2021. https://doi.org/10.1038/s41386-021-01015-4.

3. McLellan AT, Lewis DC, O'brien CP, Kleber HD. Drug dependence, a chronic medical illness: implications for treatment, insurance, and outcomes evaluation. JAMA. 2000;284:1689-95.

4. Siegel RL, Miller KD, Fuchs HE, Jemal A. Cancer statistics, 2021. CA Cancer J Clin. 2021;71:7-33.

5. Memon A, Bannister P, Rogers I, Sundin J, Al-Ayadhy B, James PW, et al. Changing epidemiology and age-specific incidence of cutaneous malignant melanoma in England: An analysis of the national cancer registration data by age, gender and anatomical site, 1981-2018. The Lancet Regional Health. Lancet Reg Health Eur. 2021;2:100024. 